\title{
Highly deformable actuators made of dielectric elastomers clamped by rigid rings
}

\section{Citation}

Lu, Tongqing, Choon Chiang Foo, Jiangshui Huang, Jian Zhu, and Zhigang Suo. 2014. "Highly Deformable Actuators Made of Dielectric Elastomers Clamped by Rigid Rings." Journal of Applied Physics 115 (18) (May 14): 184105. doi:10.1063/1.4876722.

\section{Published Version}

doi: $10.1063 / 1.4876722$

\section{Permanent link}

http://nrs.harvard.edu/urn-3:HUL.InstRepos:13919155

\section{Terms of Use}

This article was downloaded from Harvard University's DASH repository, and is made available under the terms and conditions applicable to Open Access Policy Articles, as set forth at http:// nrs.harvard.edu/urn-3:HUL.InstRepos:dash.current.terms-of-use\#OAP

\section{Share Your Story}

The Harvard community has made this article openly available.

Please share how this access benefits you. Submit a story.

Accessibility 


\title{
Highly deformable actuators made of dielectric elastomers clamped by rigid rings
}

Tongqing $\mathrm{Lu}^{1,2}$, Choon Chiang $\mathrm{Foo}^{2,3}$, Jiangshui Huang², Jian Zhu4, Zhigang Suo ${ }^{2, \mathrm{a})}$

${ }^{1}$ State Key Lab for Strength and Vibration of Mechanical Structures, International Center for Applied Mechanics, School of Aerospace Engineering, Xi'an Jiaotong University, 710049 Xi'an, China

${ }^{2}$ School of Engineering and Applied Sciences, Kavli Institute for Nanobio Science and Technology, Harvard University, Cambridge, MA 02138, USA

3Institute of High Performance Computing, 1 Fusionopolis Way, \#16-16 Connexis, Singapore 138632, Singapore

4Department of Mechanical Engineering, National University of Singapore, 9 Engineering Drive 1, 117576,Singapore

\begin{abstract}
In the nascent field of soft machines, soft materials are used to create devices that actuate robots, sense environment, monitor health, and harvest energy. The soft materials undergo large deformation in response to external stimuli, often leading to instability that is usually undesirable but sometimes useful. Here we study a dielectric elastomer membrane sandwiched between two soft conductors, rolled into a hollow tube, pre-stretched in the hoop direction, and fixed at the ends of the tube to two rigid rings. This structure functions as an electromechanical transducer when the two rings are subject to a mechanical force and the two conductors are subject to an electrical voltage. We formulate a computational model by using a variational principle, and calculate the large and inhomogeneous deformation by solving a nonlinear boundary-value problem. We demonstrate that large actuation strains are achievable when the height-to-radius ratio of the tube is small and the hoop pre-stretch is large. The model provides a tool to analyze various modes of instability and optimize the electromechanical performance.
\end{abstract}

Keywords: dielectric elastomers, electromchanical transducer, inhomogeneous deformation

a)Corresponding author, email: suo@seas.harvard.edu 


\section{Introduction}

A membrane of dielectric elastomer deforms in response to an applied voltage. This phenomenon has been studied intensely in developing highly deformable electromechanical transducers. ${ }^{1,2}$ Attributes of these transducers include large deformation, fast response, light weight, silent operation and low cost. $3^{-6}$ Applications are wide ranging, including actuators for soft robots and MEMS,7-10 tunable lenses and haptic interfaces for mobile phones, ${ }^{11-13}$ and generators for harvesting energy from human motion and ocean waves. ${ }^{14,15}$

Here we investigate a tubular configuration (Fig. 1). A membrane of a dielectric elastomer is sandwiched between two soft conductors of negligible stiffness, rolled into a hollow tube, pre-stretched in the hoop direction, and fixed at the ends of the tube to two rigid rings. A constant mechanical force pulls one ring relative to the other. When a voltage is applied to the two conductors, electric charges of the opposite polarities spread on the two faces of the dielectric membrane, causing the membrane to reduce thickness and expands area. The tube elongates in the axial direction, and functions as an actuator. On the other hand, for pre-charged membrane, when the applied force is removed in an open-circuit condition, the membrane increase its thickness and reduces its area, boosting the voltage between the two conductors. ${ }^{16}$ In this setup, the structure functions as a generator. This tubular geometry is easy to fabricate and use. The membrane has no free edge, which may enhance reliability.

A transducer of this type was developed as a generator to harvest energy from 
ocean waves. ${ }^{17}$ The dielectric elastomer was stretched and wrapped to form a roll with a radius $R$ of $15 \mathrm{~cm}$ and a length $L$ of $20 \mathrm{~cm}$. Several rolls are connected and placed on a buoy in ocean. As the buoy floated up and down with the ocean waves, a mass attached to the rolls cyclically stretched the dielectric elastomer. The setup was connected to an electrical circuit, and converted the mechanical energy of the ocean waves to electrical energy. The maximal electrical energy harvested was about $0.1 \mathrm{~J} / \mathrm{g}$ per cycle. Analysis of idealized generators has predicted that electrical energy can be harvested at a rate about $1 \mathrm{~J} / \mathrm{g}$ per cycle. ${ }^{18}$

Here we present a computational model to analyze large and inhomogeneous deformation of this tubular configuration. We derive the governing equations and boundary conditions using a variational principle, and describe the voltage-induced deformation by using the model of ideal dielectric elastomers. We focus on the electromechanical behavior of an actuator. Our calculations demonstrate that the height-to-radius ratio of the tube, the pre-stretch in the hoop direction and the force applied between the rings significantly affect the electromechanical behavior. We compare the numerical results to two limiting cases: an unclamped flat membrane and a clamped flat membrane.

\section{Governing equations}

The theory of elastic dielectrics has been re-examined in recent years in light of the intense development of dielectric elastomer transducers. ${ }^{19}$ Several groups have developed finite element methods for dielectric elastomer actuators. ${ }^{20-24}$ Here we use 
a variational principle to derive the equations of equilibrium, and adopt a specialized numerical method taking advantage of axisymmetric shape of the curved membranes. ${ }^{25-29}$

An actuator is made of a membrane of a dielectric elastomer sandwiched between two soft conductors of negligible stiffness. In the reference state (Fig. 1a), a tube with radius $B$, length $L$, and thickness $H$, made by rolling the membrane, is subject to no force or voltage. In the rest state (Fig. 1b), the tube is pre-stretched in the hoop direction, and attached to rigid rings on both the top and the bottom edges. The radius of the rings is $b$, so that the hoop pre-stretch is $b / B$. A constant mechanical force $P$ pulls one ring relative to the other. The separation between the two rings is $L_{p r e}$. In the actuated state (Fig. 1c), subject to a voltage $\Phi$ across the thickness of the membrane, the separation between the two rings becomes $l$. The charge on the two faces of the membrane is $\pm Q$. The deformation of the tube is assumed to be axisymmetric.

The tube undergoes inhomogeneous deformation. Let $z$ be the coordinate along the axis of symmetry, $r$ be the coordinate in radial direction, and the plane $z=0$ be the middle plane of the tube. Due to axisymmetry, we identify each material particle using a single variable: the height of the material particle $Z$ when the tube is in the reference state. In a deformed state, the same material particle moves to a point in space of coordinates $(r, z)$. The functions $r=r(Z)$ and $z=z(Z)$ fully describe the shape of the deformed actuator.

Next consider two material particles at heights $Z$ and $Z+d Z$ when the tube is in 
the reference state. In a deformed state, these two material particles move to two points in space of coordinates $(r, z)$ and $(r+d r, z+d z)$. Here $d r=r(Z+d Z)-r(Z)$ and $d z=z(Z+d Z)-z(Z)$. The set of all material particles between the two particles forms a small element. When the actuator is in the reference state, the length of the element is $d Z$, and the element is vertical. When the actuator is in the deformed state, the element elongates in the longitudinal direction by the stretch $\lambda_{1}$, and is oriented at angle $\theta$ from the radial direction. Consequently, in the deformed state, the length of the element is $\lambda_{1} d Z$, and the three segments $\lambda_{1} d Z, d z$ and $d r$ form a right triangle, giving

$$
\begin{aligned}
& \frac{d z}{d Z}=\lambda_{1} \sin \theta \\
& \frac{d r}{d Z}=\lambda_{1} \cos \theta
\end{aligned}
$$

Consider a circle of material particles at a certain height when the actuator is in the reference state. The perimeter of the circle is $2 \pi B$ in the reference state, and is $2 \pi r$ in the deformed state, so that the deformation causes a hoop stretch $\lambda_{2}=r / B$. The deformation of the membrane is inhomogeneous: the angle and the two stretches are functions of the material particles, $\theta(Z), \lambda_{1}(Z)$ and $\lambda_{2}(Z)$. The thickness of the membrane is uniform in the reference state, $H$, but is nonuniform in the deformed state, $h(Z)$. The elastomer is taken to be incompressible, so that $H=\lambda_{1} \lambda_{2} h$. Because the dielectric is sandwiched between two conductors, the voltage $\Phi$ applied between the two conductors causes a homogeneous electric potential in the dielectric. The electric field $E$, however, is inhomogeneous in the dielectric, and relates to the 
voltage by $E=\Phi / h$. The voltage spreads charges of the opposite polarities $\pm Q$ on the two faces of the dielectric. The magnitude of the charge is $Q=2 \pi \int D r d z$, where $D$ is the electric displacement.

The mechanisms applying the mechanical force and electrical voltage, together with the dielectric, constitute a thermodynamic system. We assume that the system is held at a constant temperature. Let $W$ be the Helmholtz free energy density of an element of the dielectric elastomer divided by the volume of the element in the reference state. The elastomer is taken to be incompressible, such that the thermodynamic state of the element is described by the stretches, $\lambda_{1}$ and $\lambda_{2}$, as well the electric displacement $D$. The Helmholtz free energy of this thermodynamic system is

$$
\Pi=2 \pi B H \int_{-L / 2}^{L / 2} W\left(\lambda_{1}, \lambda_{2}, D\right) d Z-P l-\Phi Q
$$

where $W\left(\lambda_{1}, \lambda_{2}, D\right)$ is the free energy density for the dielectric elastomer, $-P l$ is the potential energy of the mechanical force, and $-\Phi Q$ is the potential energy of the voltage source.

The first order variation of the free energy gives the governing equations and boundary conditions. Inspecting (1) and (2), we have $\delta \lambda_{1}=\frac{d \delta z}{d Z} \sin \theta+\frac{d \delta r}{d Z} \cos \theta$. By definition we have $\delta \lambda_{2}=\frac{\delta r}{B}$. Inserting them into the variation of (3), we obtain the governing equations:

$$
\begin{gathered}
\frac{d}{d Z}\left(s_{1} \sin \theta\right)=0 \\
\frac{d}{d Z}\left(s_{1} \cos \theta\right)-\frac{s_{2}}{B}=0 \\
\frac{\partial W\left(\lambda_{1}, \lambda_{2}, D\right)}{\partial D}=E
\end{gathered}
$$

with boundary conditions: 


$$
\begin{gathered}
\left.\left(2 \pi B H s_{1} \sin \theta-P\right) \delta z\right|_{0} ^{L / 2}=0 \\
\left.s_{2} \cos \theta \delta r\right|_{0} ^{L / 2}=0
\end{gathered}
$$

where the nominal stresses are $s_{1}=\frac{\partial W\left(\lambda_{1}, \lambda_{2}, D\right)}{\partial \lambda_{1}}-\frac{\Phi}{H} D \lambda_{2}$, $s_{2}=\frac{\partial W\left(\lambda_{1}, \lambda_{2}, D\right)}{\partial \lambda_{2}}-\frac{\Phi}{H} D \lambda_{1}$. The true stress is related to the nominal stress by $\sigma_{1}=s_{1} \lambda_{1}, \sigma_{2}=s_{2} \lambda_{2}$

We adopt the model of ideal dielectric elastomers. ${ }^{30}$ This model assumes that the electric displacement is linearly proportional to the electric field, such that $D=\varepsilon E$, and that the permittivity $\varepsilon$ is a constant independent of deformation. Integrating (6), we obtain the free energy density

$$
W\left(\lambda_{1}, \lambda_{2}, D\right)=W_{\text {stretch }}\left(\lambda_{1}, \lambda_{2}\right)+\frac{D^{2}}{2 \varepsilon}
$$

Here $W_{\text {stretch }}\left(\lambda_{1}, \lambda_{2}\right)$ is the free energy of the elastomer as a function of the stretches in the absence of the electric field.

We adopt a free energy function that captures the stretch-stiffening behavior. In an elastomer, each individual polymer chain has a finite contour length. When the elastomer is subject to no loads, the polymer chains are coiled, allowing a large number of conformations. Subject to loads, the polymer chains become less coiled. As the loads increase, the end-to-end distance of each polymer chain approaches the finite contour length, and the elastomer approaches a limiting stretch. On approaching the limiting stretch, the elastomer stiffens steeply. To account for this behavior, we adopt the Gent model: ${ }^{11}$ 


$$
W_{\text {stretch }}\left(\lambda_{1}, \lambda_{2}\right)=-\frac{\mu}{2} J_{\lim } \log \left(1-\frac{\lambda_{1}^{2}+\lambda_{2}^{2}+\lambda_{1}^{-2} \lambda_{2}^{-2}-3}{J_{\lim }}\right)
$$

where $\mu$ is the shear modulus, and $J_{\lim }$ is a material constant related to the limiting stretch. When the stretches are small, $\lambda_{1}^{2}+\lambda_{2}^{2}+\lambda_{1}^{-2} \lambda_{2}^{-2}-3 \ll<J_{\lim }$, the Gent model recovers the neo-Hookean model, $W_{\text {stretch }}\left(\lambda_{1}, \lambda_{2}\right)=(\mu / 2)\left(\lambda_{1}^{2}+\lambda_{2}^{2}+\lambda_{1}^{-2} \lambda_{2}^{-2}-3\right)$. When the stretches approach the limit, $\lambda_{1}^{2}+\lambda_{2}^{2}+\lambda_{1}^{-2} \lambda_{2}^{-2}-3 \rightarrow J_{\text {lim }}$, the Gent model stiffens steeply. In our calculations, we use $\mu$ to normalize various quantities, and assume $J_{\lim }=120$, a representative value for a widely used dielectric elastomer, $\mathrm{VHB}^{\mathrm{TM}} \cdot{ }^{32}$

\section{Numerical Simulation}

This section briefly describes a numerical method to calculate the inhomogeneous deformation. We first write the equation to express $d \theta / d Z$ explicitly by expanding (4) and (5),

$$
\frac{d \theta}{d Z}=-\frac{s_{2}}{B s_{1}} \sin \theta
$$

Combining (4) and (7), we have the algebraic equation as

$$
2 \pi B H s_{1} \sin \theta-P=0
$$

With (2), (11), and (12), we solve this boundary-value problem in the interval o $<Z<$ $L / 2$ by using the shooting method. By symmetry $\theta(0)=\pi / 2$. An initial guess is provided for $r(\mathrm{o})$, and the initial value for $\lambda_{1}(\mathrm{o})$ is solved with (12). These values are used as the initial conditions to numerically integrate the ordinary differential equations (2) and (11), together with the algebraic equation (12), to obtain the three 
functions $r(Z), \theta(Z)$, and $\lambda_{1}(Z)$. The values are solved iteratively until they satisfy the boundary condition $r(L / 2)=b$.

Once the functions $r(Z), \theta(Z)$, and $\lambda_{1}(Z)$ are determined, the separation between the two rigid rings, $l$, is determined by integrating (1) subject to the condition $z(0)=0$.

\section{Computational results and discussions}

The relative change in the height of the actuator from the rest to the actuated states defines the actuation strain (i.e., the normalized stroke):

$$
\xi_{\text {actuation }}=\frac{l}{L_{\text {pre }}}-1 .
$$

In designing this type of actuators, one may vary the mechanical force, the height-to-radius ratio of the tube, and the hoop pre-stretch of the elastomer. We represent these variables using three dimensionless parameters: $P /(2 \pi \mu B H), L / B$, and $b / B$. We first fix the axial load $P /(2 \pi \mu B H)=1$ and hoop pre-stretch $b / B=3$, and consider the effect of the aspect ratio $L / B$. The aspect ratio strongly affects the cross sections of the membrane (Fig. 2). The actuated state corresponds to the state of the maximum actuation, when loss of tension occurs which may cause the membrane to form wrinkles. The maximum axial actuation strain is around $316 \%$ for the actuator with $L / B=0.5$, but is only $15 \%$ for the actuator with $L / B=6$. We will explain the huge difference in detail later.

We next plot the distributions for the stretches, stresses, and electric field in the membrane under various levels of voltages for actuators with different aspect ratios: $L / B=0.5$ (Fig. 3) and $L / B=6$ (Fig. 4). Due to symmetry, we present results for 
the upper half of the structure. In both cases, stresses and the electric field concentrate at the clamped boundary, where the membrane is most likely to fail due to electrical breakdown. We also observe that the hoop stress $\sigma_{2}$ at the clamped boundary vanishes under increasing voltage, leading to loss of tension. The inhomogeneity in deformation, stresses, and electric field becomes more pronounced for a structure with a larger aspect ratio. For example, with an aspect ratio $L / B=6$, the electric field at the top edge $(Z=L / 2)$ is approximately 8 times greater than that in the middle $(Z=0)$. For $L / B=0.5$, this difference in electric field is less than $10 \%$.

The voltage-stroke curve depends on the aspect ratio of the actuator (Fig. 5). Calculations are terminated when the loss of tension occurs. For a fixed axial force and hoop pre-stretch, the actuation strain decreases as the aspect ratio $L / B$ increases. We also include the voltage-actuation curves for two limiting conditions: an unclamped membrane under constant uniaxial tension ${ }^{32,33}$ and a clamped membrane subject to constant tension. ${ }^{34}$ The voltage-actuation curves for the tubular actuator with different aspect ratios lie between the curves of the two limiting cases. This behavior is understood as follows. For an actuator with a large aspect ratio, most parts are in an uniform uniaxial stress state. It is known that the voltage-induced actuation for dielectric elastomers under uniaxial tension is small.32,33 In the cases considered here, the actuation is even smaller than the ideal uniaxial force condition, due to inhomogeneity and loss of tension at the clamped boundaries. On the other hand, an actuator with a small aspect ratio approaches the condition of a clamped membrane subject to constant tension. Experiments have shown that large actuation 
is achievable for such clamped membranes. ${ }^{34}$ Therefore, by decreasing the aspect ratio, the loading condition changes from an unclamped case to a clamped case, with a corresponding increase in actuation.

The voltage-stroke curve also depends on the applied force $P$ (Fig.6) and pre-stretch $b / B$ (Fig. 7). For a prescribed aspect ratio $L / B$ and pre-stretch $b / B$, the actuation strain decreases with increasing $P$. This behavior implies that under greater $P$, the structure behaves more like an unclamped membrane under uniaxial tension. Conversely, for a prescribed aspect ratio and pre-load, the actuation strain increases with increasing pre-stretch in the hoop direction. In this case, the structure behaves more like a clamped membrane under constant tension. The pre-stretch effects for this actuator will not be discussed in detail since they are similar to those in the two limiting cases.

In designing the dielectric elastomer for applications such as actuators and generators, a large deformation range may be one of the most important design goals. As discussed above, the deformation range of the structure increases when the aspect ratio $L / B$ decreases, such that the structure approaches the clamped membrane condition. However, under such loading conditions, the mass of the entire structure may increase substantially due to the rigid rings needed to constrain the elastomer at the top and bottom edges. Thus the overall efficiency and specific energy density of the whole structure may decrease. The optimized performance would be a trade-off between the deformation range and specific energy density, which would depend on the material and loading parameters as illustrated in Figs. 5-7. A recent design where 
the hoop direction is constrained by stiff thin fibers might be an optional choice. ${ }^{6}$ We also note that in our current calculations, loss of tension at the boundary (Figs. 3 and 4) precedes electrical breakdown, when we assume the dielectric breakdown strength to be $200 \mathrm{MV} / \mathrm{m} \cdot{ }^{32,35}$ Thus, electrical breakdown of the dielectric elastomer is not discussed.

\section{Conclusions}

We present a computational model for the tubular transducers. We calculate the inhomogeneous deformation by formulating a nonlinear boundary-value problem. We demonstrate that the actuation is significantly affected by the height-to-radius ratio of the tube and the loading conditions. These effects are interpreted by comparing with two ideal limiting cases. A short tube approaches the case of a clamped flat membrane under constant tension, and large actuation strain is achievable. A long tube approaches the case of an unclamped membrane under uniaxial tension, and the actuation strain becomes small. Inhomogeneous fields of various quantities are also analyzed. Optimization of these actuators should combine the consideration of deformation range and energy density. These results will help to design such tubular transducers.

\section{Acknowledgements}

The work at Harvard was supported by NSF MRSEC (DMR-0820484). The work at XJTU was supported by NSFC (No. 11321062). Lu was supported by China 
Scholarship Council as a visiting scholar for two years at Harvard University. CC Foo gratefully acknowledges support from A*STAR PDF and SERC Grant (132-183-0025).

J Zhu was supported by MOE Tier 1 (R-265-000-444-133). ZS acknowledges a visiting appointment at the International Center for Applied Mechanics.

\section{References}

1. R. Pelrine, R. Kornbluh, Q. B. Pei and J. Joseph, Science 287, 836 (2000).

2. F. Carpi, S. Bauer and D. De Rossi, Science 330, 1759 (2010).

3. P. Brochu and Q. B. Pei, Macromol. Rapid. Commun. 31, 10 (2010).

4. I. A. Anderson, T. A. Gisby, T. McKay, B.M. O’Brien and E. Calius, J. Appl. Phys. 112, 041101 (2012).

5. R. Kornbluh, pp. 79-90 in Dielectric Elastomers as Electromechanical Transducers, edited by F. Carpi, D. D. Rossi, R. Kornbluh, R. Pelrine and P. S. Larsen. Elsevier, Oxford (2008).

6. R. Kornbluh, R. Pelrine, H. Prahlad, A. Wong-Foy, B. McCoy, S. Kim, J. Eckerle, and T. Low, MRS Bulletin 37, 246 (2012).

7. Q. B. Pei, R. Pelrine, S. Stanford, R. Kornbluh and M. Rosenthal, Synthetic Metals. 135, 129 (2003).

8. R. Shankar, T. K. Ghosh and R. J. Spontak, Adv. Mater. 19, 2218 (2007).

9. G. Kovacs, L. Düring, S. Michel and G. Terrasi, Sens. Actuators A 155, 299 (2009).

10. S. Akbari and H.R. Shea, J. Micromech. Microeng. 22, 045020 (2012).

11. F. Carpi, G. Frediani, S. Turco and D. De Rossi, Adv. Func. Mater. 21, 4002 (2011).

12. S. Shian, R. M. Diebold, and D. R. Clarke, Opt. Express 21, 8669 (2013).

13. A. Cheng, WW-EAP Newsletter 13, 2 (2011).

14. T. Mckay, B. M. O'Brien, E. Calius and I. A. Anderson, Appl. Phys. Lett. 97, 
$062911(2010)$.

15. R. Kaltseis, C. Keplinger, R. Baumgartner, M. Kaltenbrunner, T.F. Li, P. Mächler, R. Schwödiauer, Z. G. Suo and S. Bauer, Appl. Phys. Lett. 99, 162904 (2011).

16. S. J. A. Koh, X. H. Zhao, and Z. G. Suo, Appl. Phys. Lett. 94, 262902 (2009).

17. R. Kornbluh, R. Pelrine, H. Prahlad, A. Wong-Foy, B. McCoy, S. Kim, J. Eckerle, and T. Low, Proc. SPIE, 797605 (2011), DOI:10.1117/12.882367.

18. S. J. A. Koh, C. Keplinger, T. F. Li, S. Bauer, and Z. G. Suo, IEEE/ASME Trans. Mechatro. 16, 33 (2011).

19. Z.G. Suo, Acta Mechanica Solida Sinica 23, 549 (2010).

20. D. K. Vu, P. Steinmann, and G. Possart, Int. J. Numer. Methods Eng. 7o, 685 (2007).

21. X. H. Zhao, and Z. G. Suo, Appl. Phys. Lett. 93, 251902 (2008).

22. B. O’Brien, T. McKay, E. Calius, S. Xie, and I. Anderson, Appl. Phys. A-Mater. 94, 507 (2009).

23. H. S. Park, Z. G. Suo, J. X. Zhou, and P. A. Klein, Int. J. Solids. Struct. 49, 2187 (2012).

24. D. L. Henann, S. A. Chester, and K. Bertoldi, J. Mech. Phys. Solids 61, 2047 (2013).

25. N. Goulbourne, E. Mockensturm, and M. Frecker, J. Appl. Mech. Trans. ASME 72, 899 (2005).

26. T. Q. Lu, S. Q. Cai, H. M. Wang, and Z. G. Suo, J. Appl. Phys. 114, 104104 (2013).

27. T. H. He, L. L. Cui, C. Chen and Z. G. Suo, Smart Mater. Struct. 19, o85017 (2010).

28. H. M. Wang, S. Q. Cai, F. Carpi, and Z. G. Suo. ASME J. Appl. Mech. 79, 031008 (2012).

29. T. F. Li, C. Keplinger, R. Baumgartner, S. Bauer, W. Yang, and Z. G. Suo, J. Mech. Phys. Solids 61, 611 (2013).

3o. X. H. Zhao, W. Hong, and Z. G. Suo, Phys. Rev. B 76, 134113 (2007).

31. A.N. Gent, Rubber. Chem. Tech. 69, 59 (1996). 
T. Q. Lu, J. S. Huang, C. Jordi, G. Kovacs, R. Huang, D. R. Clarke, and Z. G. Suo. Soft Matter 8, 6167 (2012).

33. R. Huang, and Z. G. Suo. Proc. Royal Society A 468, 1014 (2012)

34. M. Kollosche, J. Zhu, Z.G. Suo, and G. Kofod. Phys. Rev. E 85, 051801 (2012)

35. G. Kofod, R. Kornbluh, R. Pelrine and P. S. Larsen, J. Intel. Mater. Sys. Struct. 14, 787 (2003).

36. J. S. Huang, T. Q. Lu, J. Zhu, D. R. Clarke, and Z. G. Suo, Appl. Phys. Lett. 1oo, 211901 (2012).

\section{Figure Captions}

Fig. 1. A roll actuator in several states. (a) In the reference state, a membrane of dielectric elastomer is in a cylindrical shape, its inside and outside faces are coated with soft conductors, and the membrane is subject to no force or voltage. (b) In the rest state, the membrane is stretched in the hoop direction, fixed to rigid rings at the two ends, and loaded by an axial force. (c) In the actuated state, the membrane is subject to a voltage across its thickness.

Fig. 2. Calculated shapes of the rest state and the actuated state with two aspect ratios:

(a) $L / B=0.5$ and (b) $L / B=6$. The pre-stretch and the pre-load for both (a) and (b) are $b / B=3$ and $P /(2 \pi u B H)=1$, respectively. The actuated state shows the maximal actuation before loss of tension.

Fig. 3 Various quantities in an actuator with aspect ratio $L / B=0.5$, pre-stretch $b / B=3$, and pre-load $P /(2 \pi \mu B H)=1$ : (a) axial stretch, (b) hoop stretch, (c) axial 
stress, (d) hoop stress, and (e) electric field.

Fig. 4 Various quantities in an actuator with aspect ratio $L / B=6$, pre-stretch $b / B=3$, and pre-load $P /(2 \pi \mu B H)=1$ : (a) axial stretch, (b) hoop stretch, (c) axial stress, (d) hoop stress, and (e) electric field.

Fig. 5 Voltage-actuation curves for actuators with different aspect ratios $L / B$. For given pre-load and hoop pre-stretch, the actuation strain decreases as $L / B$ increases. The voltage-actuation curves lie between the curves of the two limiting cases: an unclamped membrane under uniaxial tension and a clamped membrane under constant tension.

Fig. 6 Voltage-actuation curves for actuators with different pre-loads $P$. For given aspect ratio and hoop pre-stretch, the actuation strain decreases as $P$ increases.

Fig. 7 Voltage-actuation curves for actuators with hoop pre-stretch $b / B$. For given aspect ratio and pre-load, the actuation strain increases as the pre-stretch $b / B$ increases. 


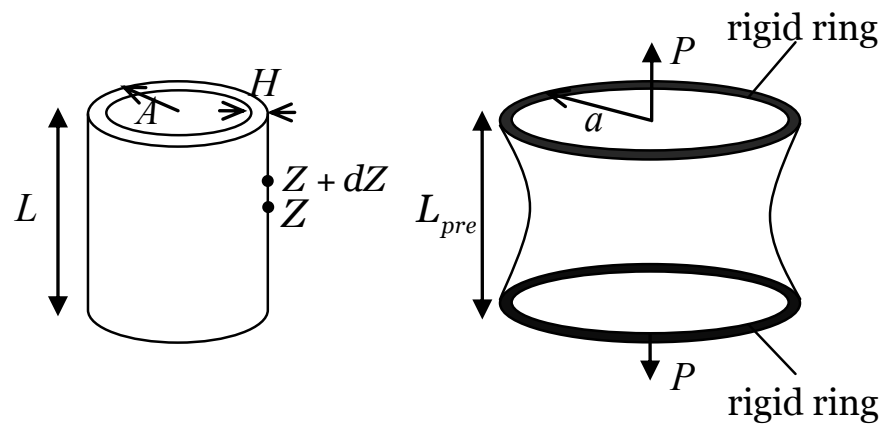

(a) reference state

(b) rest state

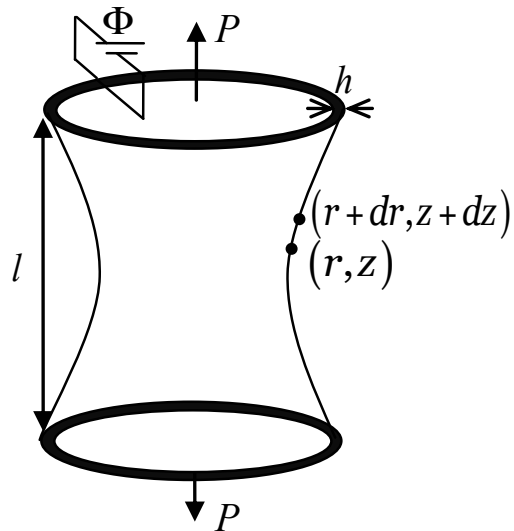

(c) actuated state

Fig. 1. A roll actuator in several states. (a) In the reference state, a membrane of dielectric elastomer is in a cylindrical shape, its inside and outside faces are coated with soft conductors, and the membrane is subject to no force or voltage. (b) In the rest state, the membrane is stretched in the hoop direction, fixed to rigid rings at the two ends, and loaded by an axial force.

(c) In the actuated state, the membrane is subject to a voltage across its thickness. 


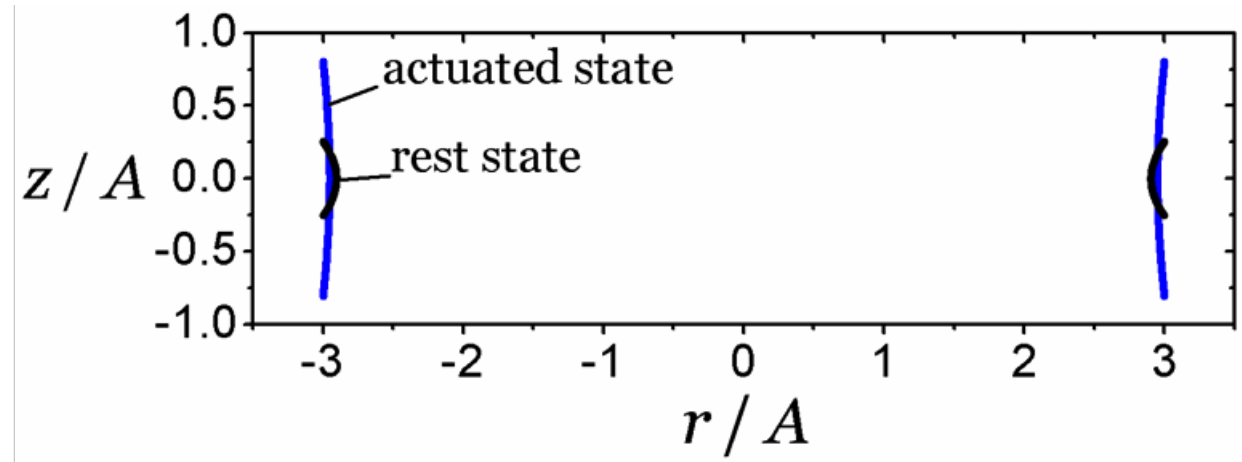

(a)

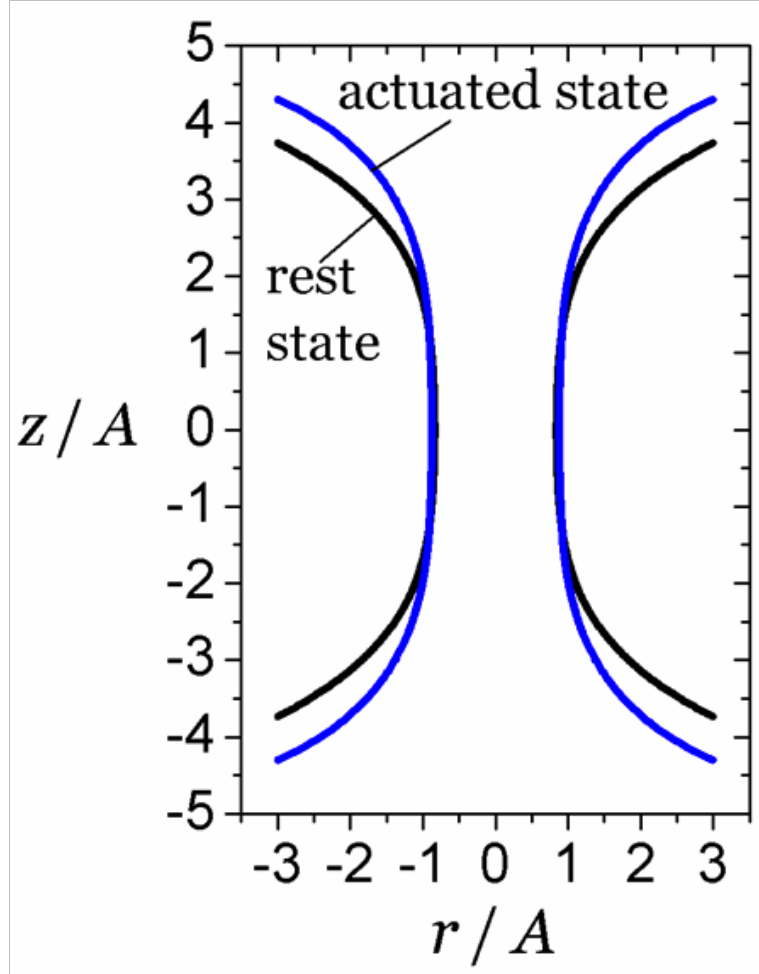

(b)

Fig. 2. Calculated shapes of the rest state and the actuated state with two aspect ratios: (a) $L / A=0.5$ and (b) $L / A=6$. The pre-stretch and the pre-load for both (a) and (b) are $a / A=3$ and $P /(2 \pi A H)=1$, respectively. The actuated state shows the maximal actuation before loss of tension. 


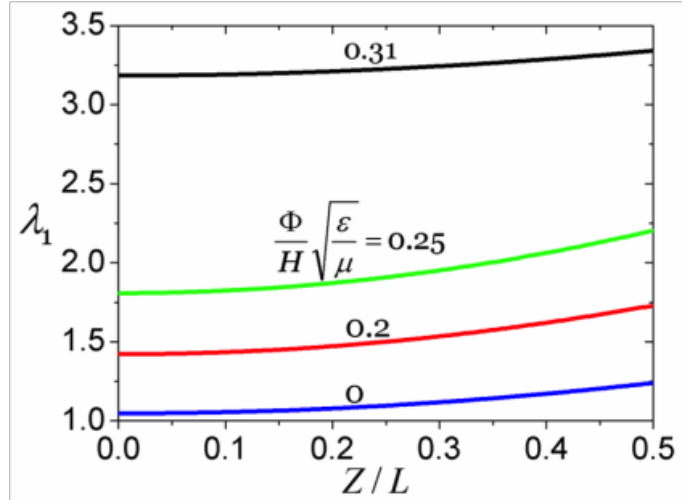

(a)

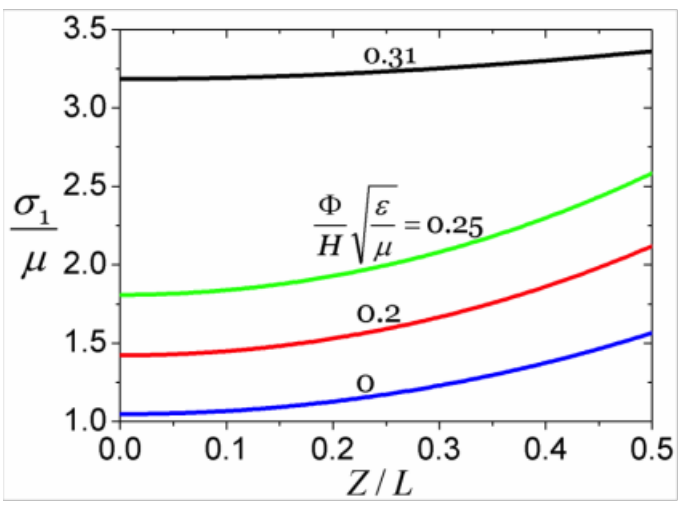

(c)

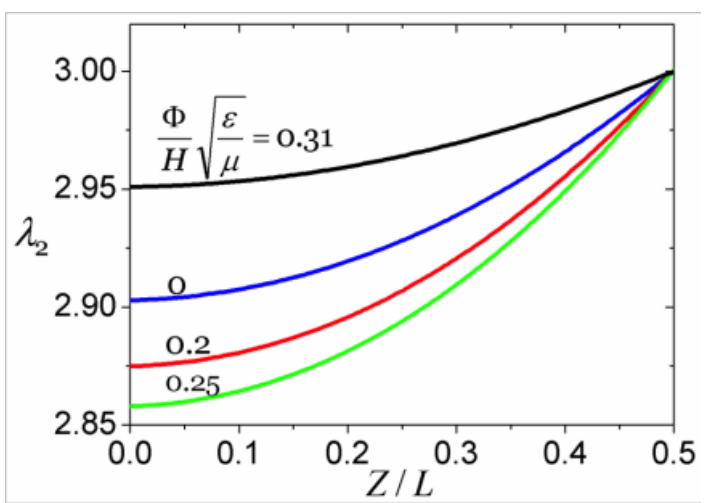

(b)

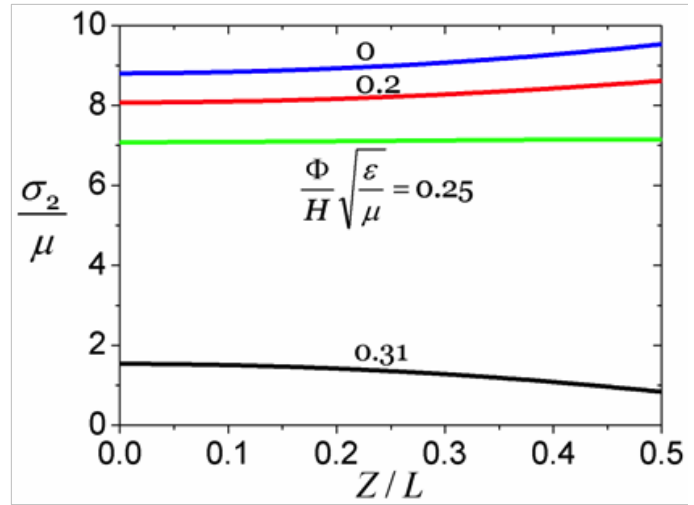

(d)

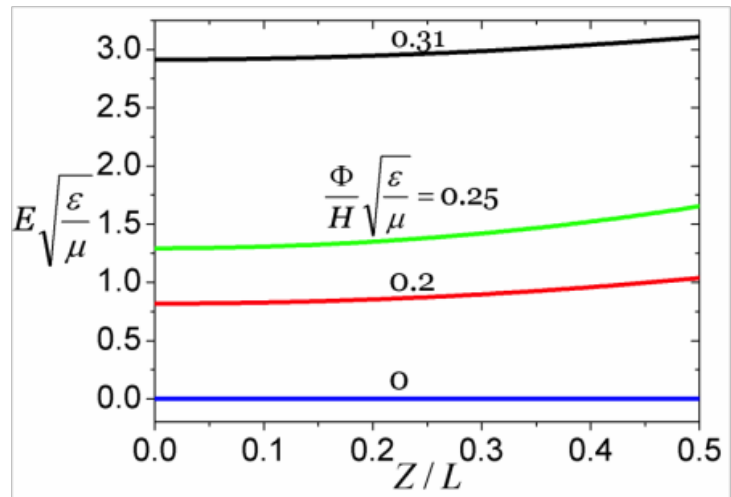

(e)

Fig. 3 Various quantities in an actuator with aspect ratio $L / A=0.5$, pre-stretch $a / A=3$, and pre-load $P /(2 \pi A H)=1$ : (a) axial stretch, (b) hoop stretch, (c) axial stress, (d) hoop stress, and (e) electric field. 


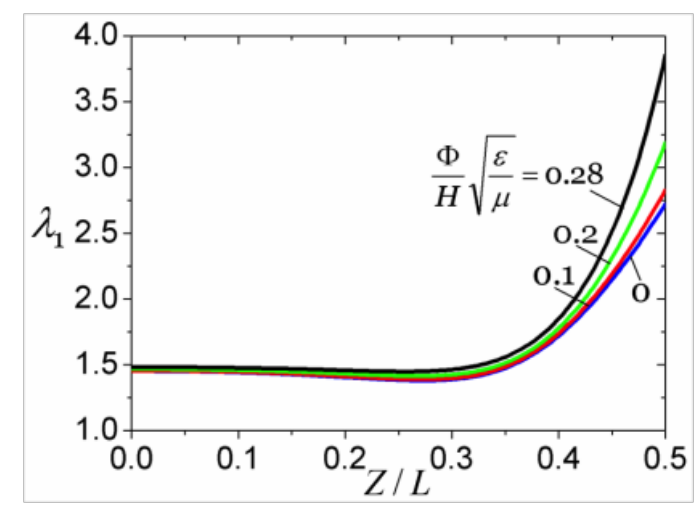

(a)

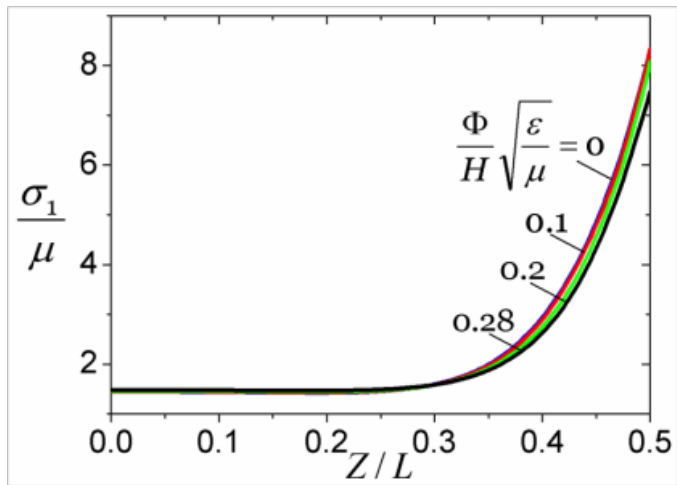

(c)

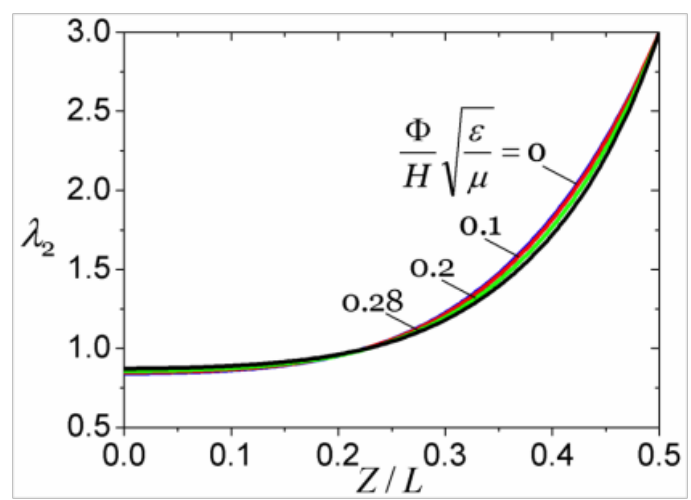

(b)

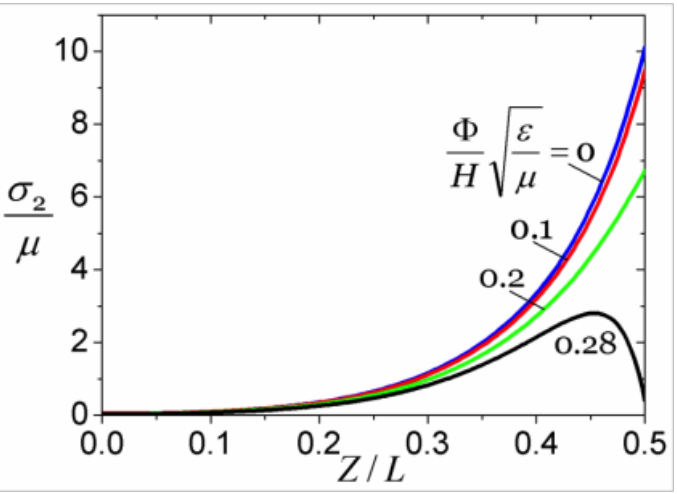

$(d)$

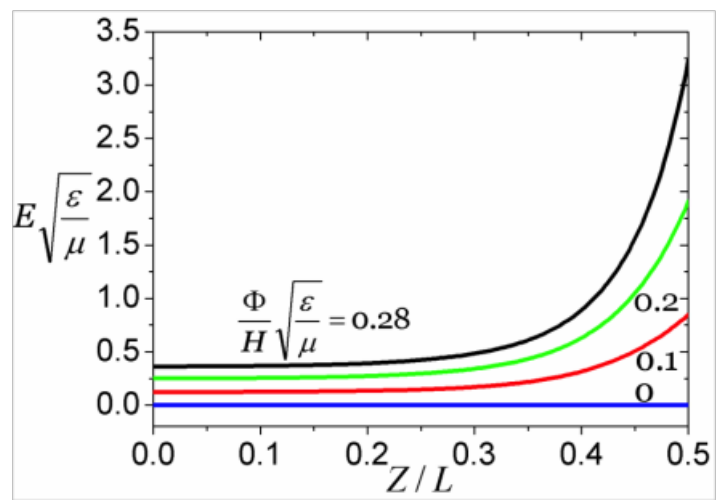

(e)

Fig. 4 Various quantities in an actuator with aspect ratio $L / A=6$, pre-stretch $a / A=3$, and pre-load $P /(2 \pi A H)=1$ : (a) axial stretch, (b) hoop stretch, (c) axial stress, (d) hoop stress, and (e) electric field. 


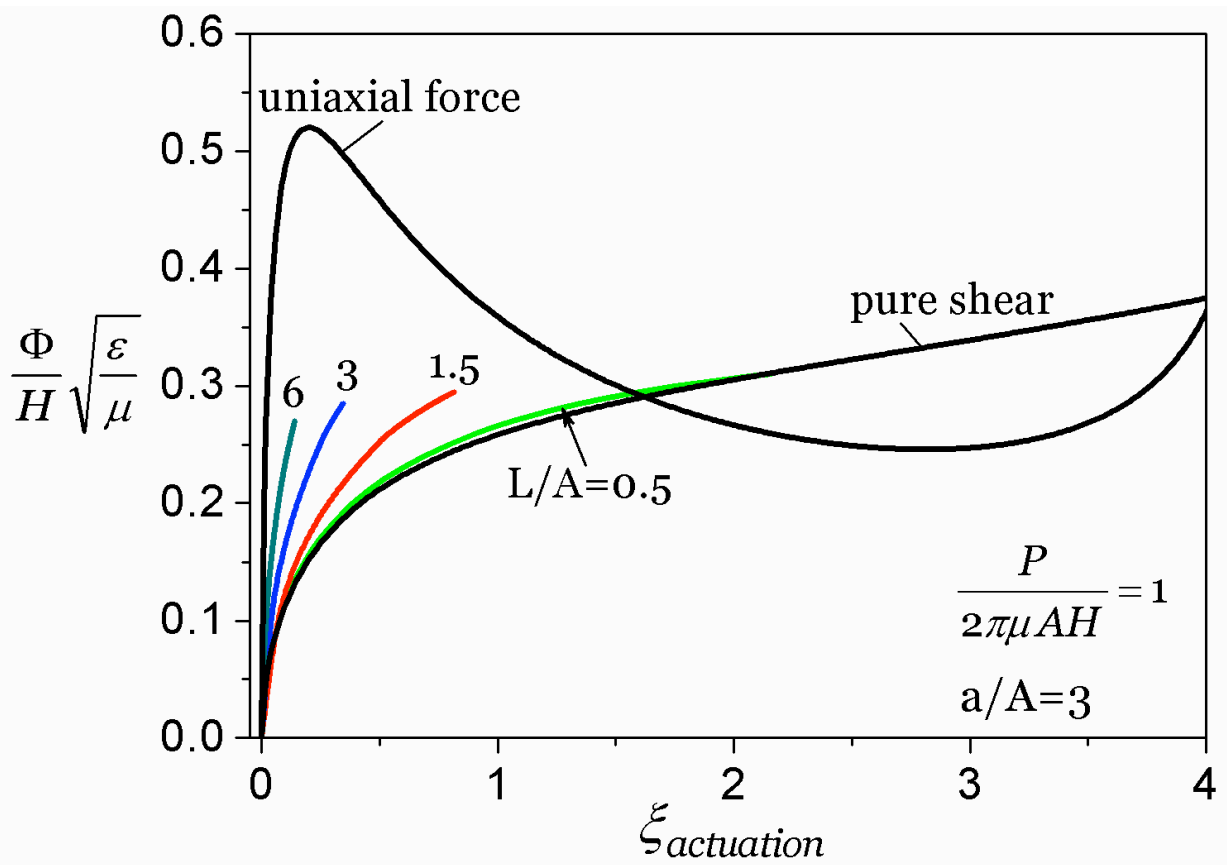

Fig. 5 Voltage-actuation curves for actuators with different aspect ratios $L / A$. For given pre-load and hoop pre-stretch, the actuation strain decreases as $L / A$ increases. The voltage-actuation curves lie between the curves of the two limiting cases: an unclamped membrane under uniaxial tension and a clamped membrane under constant tension. 


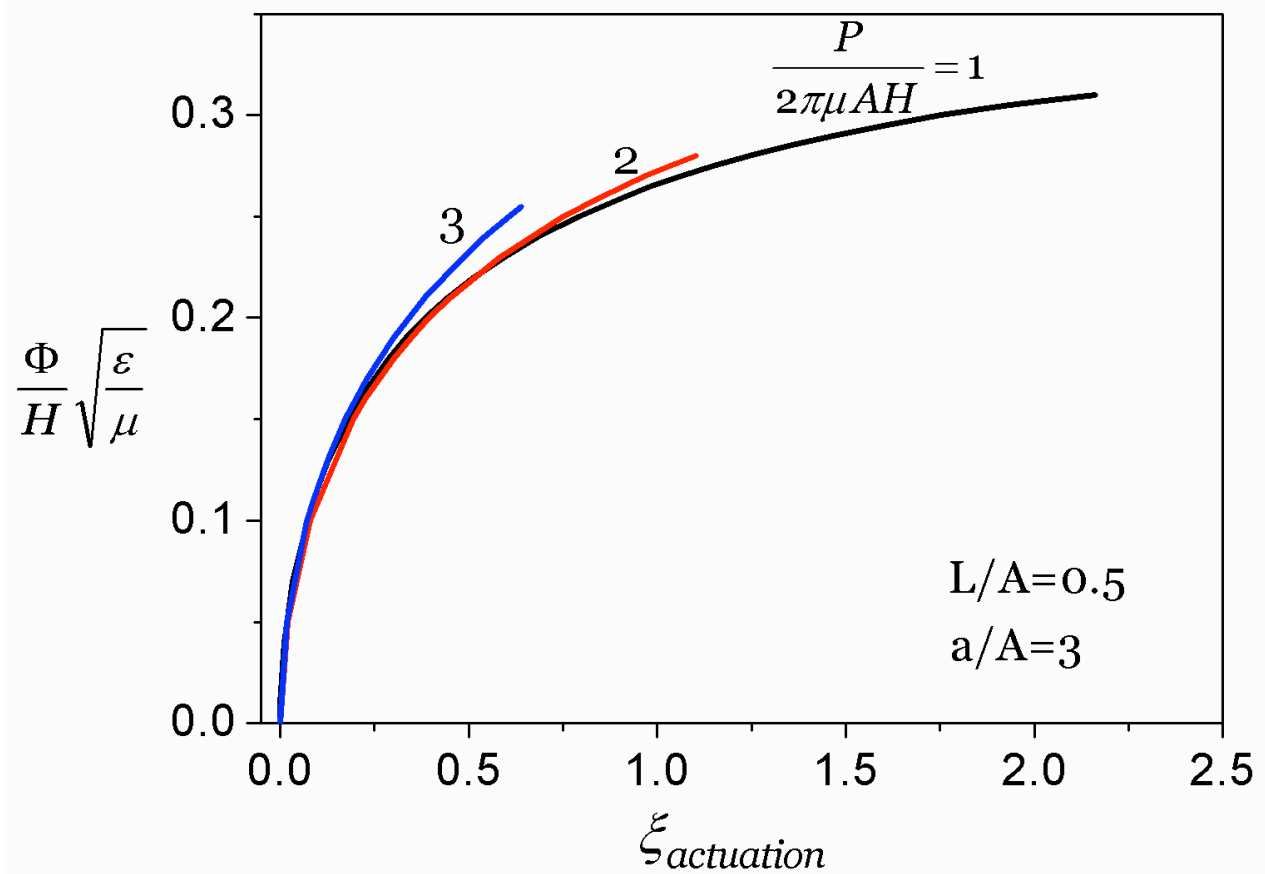

Fig. 6 Voltage-actuation curves for actuators with different pre-loads $P$. For given aspect ratio and hoop pre-stretch, the actuation strain decreases as $P$ increases. 


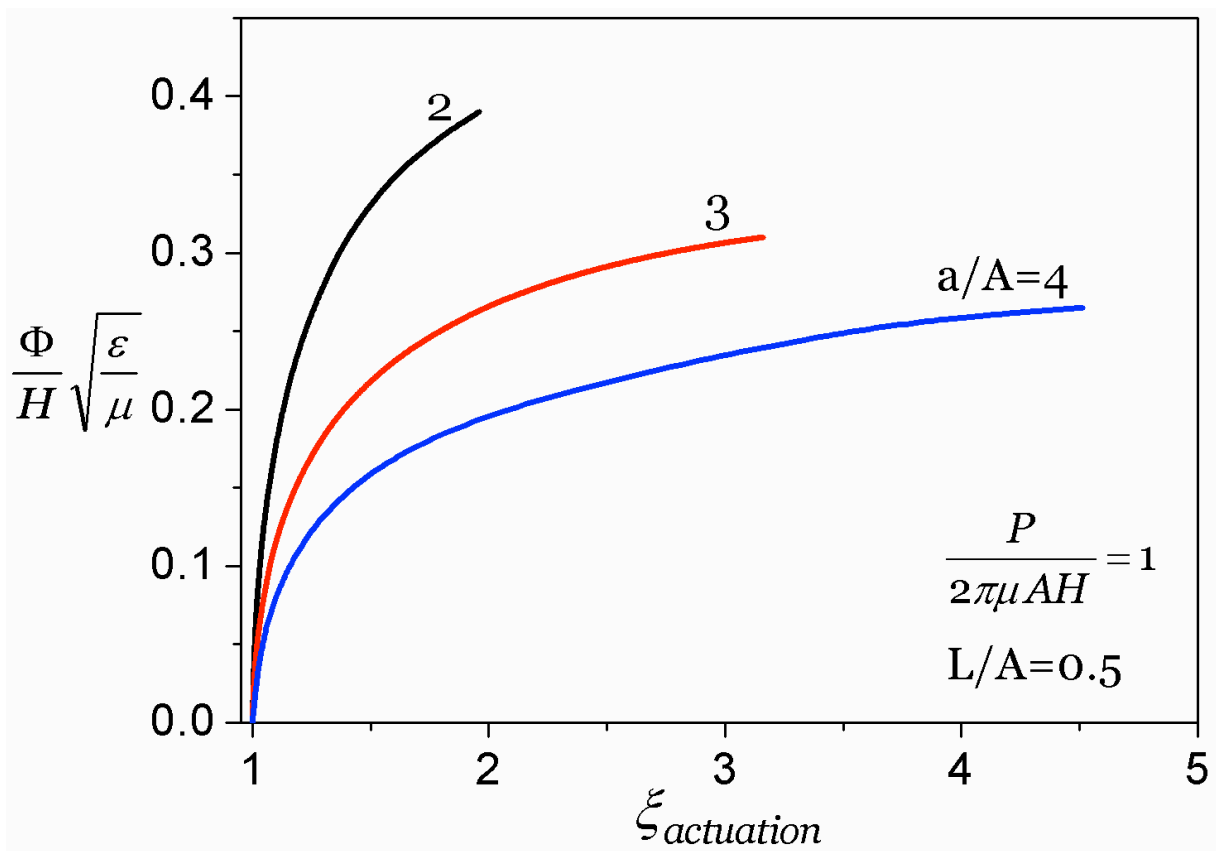

Fig. 7 Voltage-actuation curves for actuators with hoop pre-stretch $a / A$. For given aspect ratio and pre-load, the actuation strain increases as the pre-stretch $a / A$ increases. 